\title{
ACTUALITE
}

\section{La fièvre aphteuse en Asie du Sud-Est Situation et plan de lutte}

Dans toutes les régions du monde, la fièvre aphteuse constitue de nos jours l'une des principales menaces, sinon la plus importante, pour l'élevage bovin. Aussi, les autorités vétérinaires nationales et les organisations internationales telles que l'OIE' et la FAO2 considèrent-elles comme prioritaires la prévention et la lutte contre cette affection.

En Asie du Sud-Est, la fièvre aphteuse est un sujet de préoccupation dans tous les pays et la $\mathrm{FAO} / \mathrm{APHCA} \mathrm{A}^{3}$ a fourni des efforts ininterrompus pour lutter contre la maladie, depuis son apparition en 1976. Plusieurs missions ont été organisées sur place et un examen général de la situation ainsi que des recommandations ont été publiés en $1984^{4}$. Une proposition a été présentée en vue d'organiser une campagne internationale en Asie, dans la région de I'APHCA, mais celle-ci ne s'est pas encore matérialisée.

Bien que des progrès sensibles aient été réalisés dans certains pays insulaires de l'Asie du Sud-Est 5 tels que l'Indonésie, la plupart des pays asiatiques qui ont des frontières communes continuent de subir les conséquences de l'importation d'animaux contaminés. Les conflits civils et militaires, en Indochine par exemple, ont entraîné l'interruption, pendant plusieurs années, des programmes de prophylaxie dans la sous-région.

Alors que le contexte politique et économique commençait à s'améliorer, les déplacements légaux et illégaux d'animaux d'un pays à l'autre se sont considérablement accrus et la situation de la fièvre aphteuse s'est aggravée dans certains pays du Sud-Est asiatique.

C'est dans ces circonstances que s'est tenu à Pattaya, en Thaillande, en novembre 1990, le symposium de l'OIE consacré à la lutte contre les principales maladies du bétail en Asie et qu'a été discutée la stratégie de la prophylaxie anti-aphteuse en Asie du Sud-Est. Au cours du symposium, il a été recommandé que l'OIE crée une sous-commission pour la lutte contre la fièvre

1. OIE : Office international des Épizooties.

2. FAO : Food and Agriculture Organization of the United Nations - Organisation des Nations Unies pour l'alimentation et l'agriculture.

3. APHCA : Regional Animal Production and Health Commission for Asia, the Far East and the SouthWest, Pacific - Commission régionale de la production et de la santé animales pour l'Asie et le Pacifique.

4. Rapport du Groupe de travail FAO/APHCA sur la fièvre aphteuse et sa prophylaxie dans la région de l'APHCA (Bangkok, 3-8 octobre 1983). Publié en 1984.

5. L'Asie du Sud-Est regroupe les pays de l'Indochine (Cambodge, Laos, Malaysia, Myanmar, Singapour, Thaïlande et Viêt-nam) et de l'Insulinde (Brunéi, Indonésie et Philippines). Seuls sept de ces pays sont intéressés par le plan de lutte contre la fièvre aphteuse. 
aphteuse en Asie du Sud-Est, dans le but de promouvoir dans la sous-région des campagnes faisant l'objet d'une coordination internationale.

Cette recommandation a été approuvée par le Comité international de l'OIE en 1991 et la première réunion du Groupe de coordination pour la lutte contre la fièvre aphteuse en Asie du Sud-Est s'est déroulée en février 1992, à Bangkok (Thaillande), dans le cadre du programme du fonds OIE/Japon et avec la participation de la FAO.

La seconde réunion du même Groupe s'est tenue en février 1993. Le Cambodge, le Laos, la Malaysia, le Myanmar, les Philippines, la Thaillande et le Viêt-nam étaient représentés. Les participants ont décidé qu'une stratégie globale commune devrait être suivie par ces pays pour la prophylaxie et/ou l'éradication de la maladie. Ils sont également convenus de mettre au point des plans nationaux reposant sur la stratégie fixée. Ces plans ont été discutés au cours de la troisième réunion du Groupe de coordination qui s'est tenue en Malaysia en février 1994.

\section{L'élevage dans la région}

Le secteur de l'élevage en Asie du Sud-Est est principalement constitué de petits exploitants qui élèvent plusieurs espèces en petit nombre. L'objectif est essentiellement de subvenir aux besoins familiaux, d'élever des animaux de trait et, parfois, de revendre du bétail. II existe également un secteur semi-industriel à vocation totalement commerciale dont l'expansion très rapide s'explique par l'amélioration sensible de la situation économique. C'est pourquoi les systèmes d'élevage utilisés en Asie du Sud-Est, qui sont inclus dans un environnement complexe et dynamique, présentent des interactions avec de nombreux autres systèmes à l'intérieur et à l'extérieur du pays, notamment les marchés internationaux et les systèmes de production à technologie avancée.

L'importance des cheptels concernés est présentée dans le tableau l. Le nombre exact d'animaux abattus et la quantité de viande produite ne sont pas toujours connus, mais on peut espérer que la consommation de viande, de lait et de produits laitiers augmentera rapidement à mesure que ces pays progresseront.

TABLEAU I

Populations animales sensibles à la fièvre aphteuse

(Source : Annuaire zoo-sanitaire FAO/OIE/OMS, 1992)

(unité : $\times 1000$ )

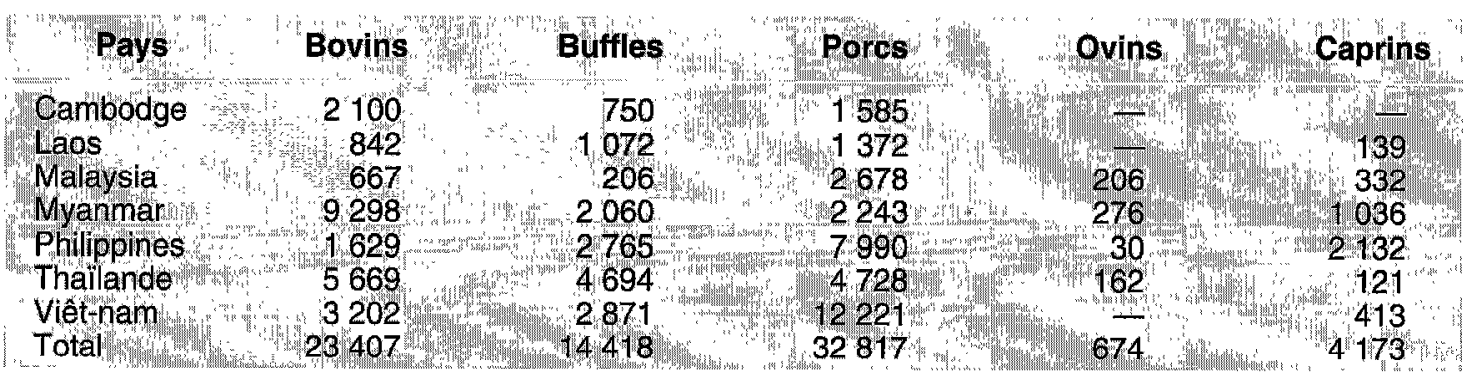




\section{Les échanges de bovins et de buffles}

Étant donné les modifications récentes de la situation politique dans la région, les déplacements internationaux s'intensifient dans plusieurs directions, en fonction de la valeur marchande des animaux et des produits qui en sont tirés. $\dot{A}$ l'exception des Philippines, tous les pays ont de grandes frontières ouvertes avec un ou plusieurs États voisins et la plupart des échanges internationaux de bétail se déroulent dans l'illégalité.

Lors de la seconde réunion du Groupe de coordination en février 1993, les représentants des différents pays ont présenté les principales tendances des échanges de bovins et de buffles dans la sous-région. On constate une multiplication des voies d'échanges internationaux, légaux et illégaux, ainsi que du nombre d'animaux commercialisés récemment, principalement en raison d'une augmentation de la demande de viande en Thaillande, en Malaysia et à Singapour.

Au tableau II figure le nombre d'animaux commercialisés en 1990, mais il n'existe aucun chiffre fiable pour les animaux commercialisés illégalement au cours de ces dernières années. Centains rapports informels indiquent que ce nombre est très supérieur à certains chiffres du tableau. Les éleveurs de la région sont cependant de plus en plus conscients du potentiel d'exportation en animaux et en produits d'origine animale vers des pays situés dans la région ou en dehors. A titre d'exemple, le Japon a importé, en 1993, 567000 tonnes de viande de boeuf désossée (à un prix variant, selon la qualité, de 3870 à 9100 \$ US la tonne) et ce tonnage était en augmentation de 34 p. 100 par rapport à 1992.

TABLEAU II

Chiffres officiels du commerce des animaux en 1990

(Source: Annuaire FAO du commerce, 1990)

(Nombre de têtes)

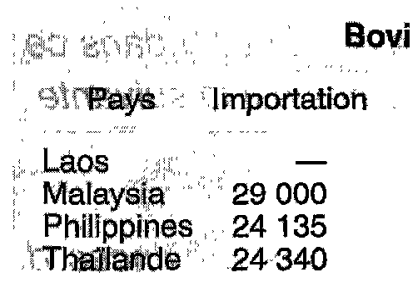

Bovins

Porcs

Ovins/caprins

Exportation
90000
500
$\frac{-}{450}$

Importation

Exportation

Importation

Exportation

$\begin{array}{rr}\text { Phillppines, } & 24135 \\ \text { Thallande } & 24340\end{array}$

450

$14 \overline{4}$
1650
1160

$\frac{580000}{30}$

${ }_{120} \frac{-}{-}$

\section{Conséquences et impact économique de la fièvre aphteuse}

La fièvre aphteuse est aujourd'hui la maladie virale la plus redoutée des éleveurs du monde entier en raison de son impact économique sur la productivité et de son effet désastreux sur le commerce des animaux et des produits d'origine animale. Elle a également des conséquences graves en raison de la perte des animaux de trait nécessaires đa la culture et au transport de différents produits. Les épisodes de fièvre aphteuse survenant pendant la saison des travaux agricoles et des récoltes posent enfin de graves problèmes aux éleveurs les plus modestes qui sont dépendants des animaux de trait. 
La maladie affecte très sérieusement les bovins, les porcs, les ovins et les caprins. Elle provoque des pertes directes car :

- elle entraîne l'avortement chez environ 25 p. 100 des femelles gravides;

- elle provoque une chute de 25 p. 100 de la production de viande lorsqu'elle est endémique ;

- elle réduit de 50 p. 100 la production laitière dans les élevages touchés ;

- elle abaisse de 25 p. 100 la production de laine dans les élevages infectés ;

- elle est associée à une mortalité d'environ 5,5 p. 100 des animaux atteints.

Au total, dans les pays touchés, elle est à l'origine d'une réduction annuelle de production d'environ 25 p. 100 par rapport à la production attendue des animaux sains.

Au décours de la maladie, les animaux peuvent présenter une surinfection chronique des lésions buccales, nasales ou podales. La déformation des onglons peut entraîner une boiterie irréversible. L'atteinte de la mamelle se traduit par une mammite chronique ou une réduction de la production laitière. II est fréquent que les animaux restent amaigris et ne reprennent pas de poids. Ce tableau est parfois associé à des lésions cardiaques. Des anomalies de l'oestrus ou de la reproduction peuvent persister pendant de longs mois. En général, les plus gros préjudices sont enregistrés dans les races de bovins et de buffles à forte productivité, par suite des pertes subies sur le lait et la viande. Les programmes de développement des élevages laitiers dans les pays de la région seront considérablement freinés par leur contamination.

\section{Situation en Asie du Sud-Est au cours des dernières années}

A mesure que se multiplient les échanges d'animaux par-delà les frontières, la vitesse de propagation et le nombre de foyers semblent s'accroître dans les pays tels que la Thaillande et la Malaysia, du fait du prix élevé du bétail dans ces pays. La situation de la fièvre aphteuse dans les pays concernés est la suivante:

\section{Cambodge}

Les populations d'animaux sensibles sont concentrées dans les provinces du Nord-Ouest et du Centre. Les principaux transferts de bovins qui influent sur la propagation de la maladie se résument comme suit :

- déplacements des provinces centrales vers celles limitrophes de la Thaïlande et du Viêt-nam et, au-delà, vers ces deux pays ;

- déplacements vers le centre du Cambodge avec les familles qui fuient les zones de conflits civils.

En 1992, les principaux épisodes de fièvre aphteuse se sont produits dans le nord-est et le centre du pays. Le type viral, déterminé par le Laboratoire mondial de référence de Pirbright était $O_{1}$. Les virus analysés au cours de ces dernières années étaient Asia-1 en 1988, $O_{1}$ en 1989, Asia-1 en 1991 et $O_{1}$ en 1992. 
Un vaccin trivalent est utilisé $\left(A_{22}, O_{1}\right.$, Asia-1) mais on comprendra que, dans de nombreux secteurs, il est difficile de vacciner les animaux. La couverture vaccinale de la population sensible est tombée à 5,5 p. 100 en 1992.

\section{Laos}

La fièvre aphteuse est endémique au Laos et entraîne des pertes économiques considérables. Le nombre de foyers a diminué en 1992 en raison des nombreuses vaccinations qui ont été effectuées. Vingt foyers de type Asia-1 ont cependant été rapportés au cours de cette année-là. Les virus présents étaient de type $A, O$ et Asia-1. Environ 50000 doses de vaccin bivalent (O, Asia-1) sont importées d'Inde chaque année. Les animaux qui pénètrent dans le pays proviennent de la République Populaire de Chine, du Viêt-nam et du Cambodge. Ils sont exportés vers la Thaillande à partir de nombreuses régions.

\section{Malaysia}

Les zones les plus préoccupantes sont les États péninsulaires contigus à la Thaillande (notamment Perlis, Kedah et Kelantan). Une zone tampon a été prévue le long de la frontière mais la couverture vaccinale a diminué au cours de ces dernières années car il était difficile de continuer à mobiliser les éleveurs en faveur de la vaccination puisque la maladie était absente depuis 1986. La Malaysia a également tenté de réduire volontairement la vaccination dans le but de déclarer la partie péninsulaire indemne de fièvre aphteuse. Des foyers ont éclaté en 1992 car il était difficile d'empêcher totalement les transferts frauduleux. La propagation consécutive a été difficile à contenir en raison de l'importance de la population animale non immunisée et de l'impossibilité d'obtenir des vaccins en quantités et au moment voulus.

Le foyer du Kelantan, dû au type $O_{1}$, a éclaté en juin 1992 ; à la mi-février 1993, 744 cas avaient été enregistrés. Les cas les plus récents ont été d'importance mineure. La vaccination est pratiquée et des contrôles routiers sont effectués à tous les points critiques, dans les États contigus au Kelantan.

L'épisode du Perlis, provoqué par le virus de type Asia-1, a débuté le 5 décembre 1992. Vingt et un foyers et 151 cas ont été dénombrés. Il a été maîtrisé à la mi-janvier 1993. La vaccination a été intensifiée (couverture de 77. $p$. 100). Les contrôles et la surveillance ont été renforcés le long des principales voies de transfert et dans les zones à risques connues.

Ces deux foyers ont été imputés à des déplacements illégaux de bovins provenant de Thailande. Il faut souligner qu'il est extrêmement difficile d'empêcher ces entrées. Les pertes économiques directes et indirectes en 1992 sont estimées à environ 1,6 million de \$ US.

\section{Myanmar}

La fièvre aphteuse reste endémique chez les bovins et les buffles mais n'a jamais été rapportée chez des porcs ou des animaux sauvages. L'incidence est maximale pendant la saison des pluies (de mai à août). On estime que 0,19 p. 100 des bovins et des buffles ont été infectés en 1991-92. Soixantehuit épisodes ont été rapportés, 21771 animaux ayant été atteints au cours 
de cette période. Les virus identifiés au Myanmar étaient de type $O$, A et Asia 1. En 1992, deux foyers de type Asia-1 ont été rapportés.

Le Myanmar a une capacité de production d'un million de doses de vaccins et il est prévu d'établir une zone tampon dans l'État des Kachins qui borde le Bangladesh.

Des déplacements d'animaux ont lieu durant presque toute l'année, sauf pendant les deux mois où se déroulent les travaux agricoles. Les déplacements se font principalement à pied d'un marché à l'autre. Des déplacements incontrôlés ont lieu à destination de la Thaillande, de la République Populaire de Chine, du Bangladesh et de l'Inde. Pratiquement aucun animal ne pénètre au Myanmar.

\section{Les Philippines}

La fièvre aphteuse est endémique dans certaines divisions administratives (Régions III, IV et $V$ de l'île de Luçon ainsi que dans la province insulaire de Masbate (Région $V)$ ). Un foyer de type $C$ a affecté des porcs d'un abattoir d'Albay, dans l'île de Luçon, en janvier 1993. Les ministres de l'Agriculture et des Forêts des pays de l'ASEAN6 ont reconnu jusqu'à présent le statut des zones déclarées indemnes de fièvre aphteuse. II s'agit des provinces du Palauan (Région IV), du Mindanao occidental (Région IX) et du Mindanao du Nord (Région X) ainsi que des provinces insulaires du Basilan, du Sulu, du Tawitawi (Région IX) et des Batanes (Région II).

\section{Thailande}

En 1992, 476 foyers de fièvre aphteuse ont été rapportés (98 de type 0, 19 de type A, 240 de type Asia-1 et 119 de type non défini). La majorité des foyers est survenue en octobre-novembre. Un foyer grave a touché les porcs dans la Région administrative 7. Pour la plupart, les bovins se trouvent dans les Régions 3 et 6 , les buffles dans les Régions 3 et 4 et les porcs dans les Régions 2, 6 et 7. Les animaux sont déplacés du Nord et du Nord-Est vers les provinces centrales et Bangkok. Certains déplacements ont lieu vers la zone péninsulaire du Sud et inversement.

La Thaïlande dispose de toutes les infrastructures nécessaires au diagnostic. Des vaccins obtenus sur des cultures cellulaires, parfois additionnés d'adjuvant huileux, sont produits dans le pays : 4,8 millions de doses de vaccins monovalents et 6,75 millions de doses de vaccins trivalents (types 0 , A et Asia-1).

\section{Viêt-nam}

La fièvre aphteuse est confinée au Viêt-nam du Sud. Deux foyers seulement (207 animaux infectés) ont été signalés en 1992. Quatre prélèvements effectués en 1992 contenaient le virus Asia-1. La maladie avait principalement touché des buffles et des bovins mais également quelques porcs et caprins. En 1993, aucun foyer n'a été enregistré. Le virus en cause était de type Asia-1. Les programmes de prophylaxie font actuellement l'objet d'une attention toute particulière et il est 
prévu d'établir une zone indemne autour de Ho Chi Minh-Ville. Les déplacements intérieurs tendent à aller du nord vers le sud ainsi que vers Ho Chi MinhVille. Des déplacements internationaux ont lieu à partir du Laos et du Cambodge. Quelques trousses ELISA ont été reçues pour le typage des virus.

\section{Production de vaccins en Asie du Sud-Est}

Seuls deux pays de la région, la Thaïlande et le Myanmar, produisent les vaccins. En Thaillande, des vaccins ont été fabriqués à Pakchong depuis la fin des années 70. À l'heure actuelle, ils sont préparés en grandes quantités dans deux unités de production qui ont une capacité d'environ 40 millions de doses. II existe trois formules différentes : vaccins trivalents A/O/Asia-1 pour bovins, vaccins monovalents pour bovins, et vaccins monovalents pour porcs .

Les quantités nécessaires pour les bovins et les buffles en Thaillande sont estimées à 22,5 millions de doses (vaccin trivalent) par an. Le gouvernement prévoit d'augmenter la capacité de production de 20 millions de doses trivalentes.

Au Myanmar, les vaccins sont produits par l'Institut de Recherche vétérinaire d'Insein. L'unité de production a été créée dans le cadre d'un projet FAO au début des années 80 . Elle a une capacité annuelle d'un million de doses mais la quantité actuellement produite est limitée.

Aucun vaccin de type $C$ n'est produit en Asie du Sud-Est et il faut s'adresser aux producteurs extérieurs.

À l'heure actuelle, la région ne dispose d'aucun laboratoire pour effectuer le contrôle de qualité des vaccins de la fièvre aphteuse importés. Le tableau III indique la quantité de vaccins nécessaires chaque année dans les différents pays pour assurer une prophylaxie efficace de la maladie chez les bovins et les buffles. Ce tableau montre que les quantités de vaccins produites en Asie du Sud-Est sont très inférieures aux besoins. Aussi, des vaccins produits hors de la région devront-ils être utilisés pour les campagnes de vaccination tant que la capacité des unités de production n’aura pas été considérablement accrue.

\section{Services vétérinaires}

Les services vétérinaires des sept pays en sont à un stade de développement différent. À l'exception d'un ou deux d'entre eux, la plupart ont besoin de ressources supplémentaires pour renforcer leur potentiel. La description détaillée figure dans les rapports de la FAO/APHCA ainsi que dans le compte rendu de l'atelier de l'ADB'/OIE sur la déclaration des maladies animales ${ }^{8}$.

Une bonne infrastructure est nécessaire à des campagnes efficaces. Elle suppose : a) une distribution adaptée des hommes et des autres ressources; b) des systèmes de transport et de communication fiables;c) des systèmes de surveillance et de déclaration des maladies ; d) des services de diagnostic

7. ADB : Asian Development Bank - Banque asiatique pour le développement.

8. Compte rendu d'un atelier régional sur la déclaration des maladies animales. Publié par l'ADB (vol. I et II), juin 1990. 
fiables et efficaces; e) le contrôle rigoureux des déplacements d'animaux ainsi que des circuits de commercialisation corrects ; f) une législation sur la santé animale; g) les fonds nécessaires aux campagnes de prophylaxie des maladies ; h) des relations entre les secteurs public et privé; i) l'existence d'une chaîne $d u$ froid et j) la disponibilité des vaccins nécessaires répondant aux normes de qualité définies par le "Manual of Standards for Diagnostic Tests and Vaccines" de l'OIE.

Besoins annuels en vaccins de la fièvre aphteuse
(estimations approximatives)

Le tableau IV indique le nombre de vétérinaires et le nombre d'auxiliaires sanitaires présents dans les pays du groupe de coordination.

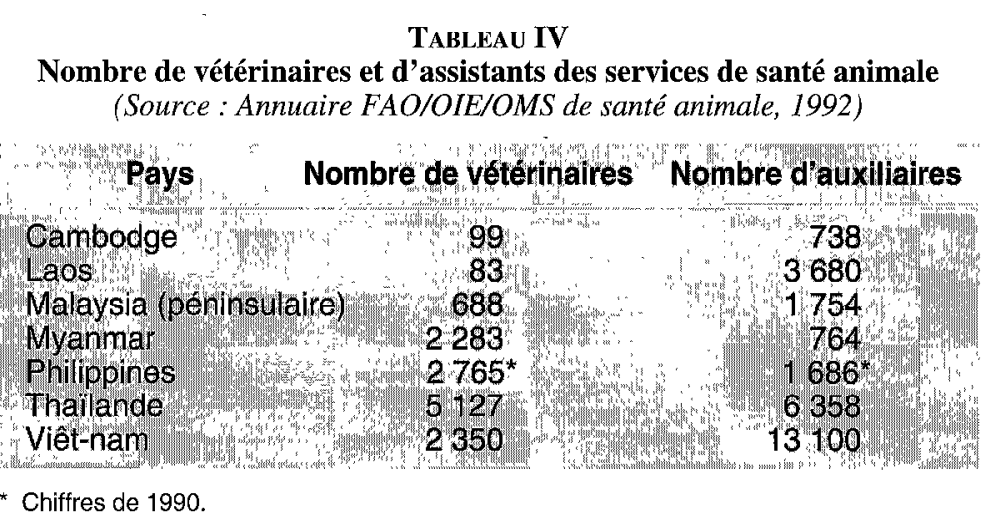

\section{Plan de lutte à long terme pour toute la région}

Un plan de lutte d'une durée de 12 ans contre la fièvre aphteuse a été décidé par les autorités sanitaires des sept pays intéressés, réunis en 1992 et 1993 à Bangkok sous l'égide de l'Office international des Epizooties. Son objectif est de renforcer l'infrastructure de base des Services vétérinaires dans les pays concernés, afin de leur permettre de lutter plus efficacement contre la maladie et d'accroître la production animale. En effet, seuls quelques pays, telles la Malaysia et la Thaillande, disposent de cette infrastructure et peuvent améliorer leur efficacité en apportant leurs ressources financières et humaines propres. Les autres États requièrent une assistance extérieure importante pour la bonne exécution du plan. A plus long terme, celui-ci vise à éradiquer la maladie de certains pays ou de certaines zones, de façon à faciliter le commerce des animaux et des produits d'origine animale dans la région. 
La stratégie retenue consiste à établir un Laboratoire régional de référence ainsi qu'un Centre de coordination régionale qui renforce la coopération entre tous les pays lors de la mise en place des opérations de surveillance épidémiologique (diagnostic) et de prophylaxie (vaccination). Celles-ci se dérouleront en trois phases :

- phase préparatoire (4 ans) : réduction du nombre de foyers,

- phase de contrôle (5 ans) : création de zones libres de la maladie,

- phase d'éradication ( 3 ans) : éradication et protection des pays ou zones libérées.

Leur exécution sera assurée avec l'appui scientifique de la Sous-commission de l'OIE pour la fièvre aphteuse et bénéficiera d'une contribution financière de plusieurs Pays membres, et notamment du Japon.

La réalisation du plan de lutte contre la fièvre aphteuse en Asie du Sud-Est, accepté par les plus hautes autorités sanitaires des pays intéressés, constitue un grand espoir pour l'élevage et l'avenir de la production animale dans. la région. L'amélioration globale de la santé animale et la disparition progressive des foyers de fièvre aphteuse dans les différents pays d'Asie du Sud-Est devraient, en effet, donner un nouvel élan au commerce international des animaux et des produits d'origine animale de cette région et contribuer à une amélioration sensible de son économie.

\section{Dr Jean Blancou}

Directeur général de l'OIE

12, rue de Prony

75017 Paris, France.
Dr Yoshihiro Ozawa

Représentant régional de l'OIE pour l'Asie et le Pacifique East 311, 1-1-1 Minamiaoyama Minato-Ku, Tokyo 107, Japon. 